Pacific Journal of Mathematic

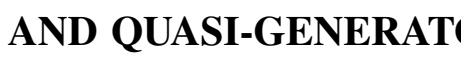




\title{
TTF CLASSES AND QUASI-GENERATORS
}

\author{
ROBERT W. MILLER
}

Let $(\mathscr{T}, \mathscr{F})$ be a hereditary torsion theory for ${ }_{A} \mathscr{M}$, the category of left $A$-modules. In this paper the property that the torsionfree class $\mathscr{F}$ be closed under homomorphic images is investigated. Particular attention is given to the case where the torsion class $\mathscr{T}$ is torsion-torsionfree (TTF). Applications to projective quasi-generators are given.

When $\mathscr{T}$ is a TTF class the question naturally arises as to when $A_{t}$, the $\mathscr{T}$-torsion submodule of $A$, is contained in a certain idempotent topologizing filter of right ideals of $A$. This condition is shown to be equivalent to the property that the torsionfree class $\mathscr{F}$ be closed under homomorphic images. Our results generalize results of Jans [6] and Bernhardt [2] characterizing the property that the torsion theory $(\mathscr{T}, \mathscr{F})$ is centrally splitting. Dropping the assumption that $\mathscr{T}$ is TTF, further investigation of the property that $\mathscr{F}$ is closed under homomorphic images yields information as to when $\mathscr{T}$ is TTF, generalizing a result due to Rutter [10]. Finally, our methods are applied to the TTF class $\mathscr{T}=\left\{{ }_{A} X \mid P \otimes{ }_{A} X=0\right\}$ where $P_{A}$ is a projective right $A$-module. The definition of $P_{A}$ being a quasi-generator is given and characterizations are obtained.

Section 2 of this paper was taken from the author's doctoral dissertation, under the direction of Professor F. L. Sandomierski, at the University of Wisconsin. Section 1 provides a generalization of the material in $\S 2$ to arbitrary TTF classes. The author expresses his gratitude to Professor Sandomierski for his guidance and encouragement.

In this paper $A$ will be an associative ring with unit and all modules will be unitary. The left (right) annihilator of $I$ in $X$ will be denoted by $l_{X}(I)\left(r_{X}(I)\right)$. It is easy to see that for a left $A$-module $X$ and a two-sided ideal $I$ of $A, r_{X}(I) \cong \operatorname{Hom}_{A}(A / I, X)$.

Dickson [4] has defined a torsion theory for ${ }_{A} \mathscr{M l}$ to be a pair $(\mathscr{T}, \mathscr{F})$ of classes of left $A$-modules satisfying

(1) $\mathscr{T} \cap \mathscr{F}=\{0\}$

(2) $\mathscr{T}$ is closed under homomorphic images.

(3) $\mathscr{F}$ is closed under submodules.

(4) For each $X \in{ }_{A} \mathscr{C l}$ there exists a (unique) submodule $X_{t} \in \mathscr{T}$ such that $X / X_{t} \in \mathscr{F}$.

A class $\mathscr{T}(\mathscr{F})$ of left modules is called a torsion (torsionfree) class provided there is a (unique) class $\mathscr{F}(\mathscr{T})$ such that $(\mathscr{T}, \mathscr{F})$ is a torsion theory. A torsion theory $(\mathscr{T}, \mathscr{F})$ is said to be hereditary if 
the torsion class $\mathscr{T}$ is closed under submodules. For further information on torsion and torsionfree classes the reader is referred to [4].

Gabriel [5] has shown that for a ring $A$ there is a one-to-one correspondence between the hereditary torsion classes of ${ }_{A} \mathscr{M}$ and the idempotent topologizing filters $F$ of left ideals of $A$ given by

$$
\mathscr{T} \longrightarrow F(\mathscr{T})=\left\{{ }_{A} I \subseteq{ }_{A} A \mid A / I \in \mathscr{T}\right\} \text {. }
$$

The inverse correspondence is given by

$$
F \longrightarrow \mathscr{T}(F)=\left\{X \in_{A} \mathscr{M} \mid l_{A}(x) \in F \text { for all } x \in X\right\} \text {. }
$$

Jans [6] has called a torsion class $\mathscr{T}$ which is also a torsionfree class for some torsion class $\mathscr{C}$, a torsion-torsionfree (TTF) class. In this case $(\mathscr{T}, \mathscr{F})$ and $(\mathscr{C}, \mathscr{T})$ are called the torsion theories associated with $\mathscr{T}$. In [6] it is shown that $\mathscr{T}$ is a TTF class if and only if $F(\mathscr{T})$ contains a unique minimal left ideal $T$. Furthermore, $T$ is an idempotent two-sided ideal, $T=A_{c}$ (the $\mathscr{C}$-torsion submodule of $A$ ), and there is a one-to-one correspondence between the TTF classes $\mathscr{T}$ of ${ }_{A} \mathscr{C}$ and the idempotent two-sided ideals of $A$ given by

$$
\mathscr{T} \longrightarrow T \text {. }
$$

The inverse correspondence is given by

$$
T \longrightarrow\left\{X \in_{A} \mathscr{C} \mid T X=0\right\} \text {. }
$$

1. TTF classes. Let $\mathscr{T} \cong{ }_{A} \mathscr{M}$ be a TTF class with associated torsion theories $(\mathscr{T}, \mathscr{F})$ and $(\mathscr{C}, \mathscr{T})$. Let $T$ be the minimal, idempotent, two-sided ideal in $F(\mathscr{T})$. One easily checks that

$$
\begin{gathered}
\mathscr{T}=\left\{{ }_{A} X \mid T X=0\right\}, \\
\mathscr{F}=\left\{{ }_{A} X \mid \operatorname{Hom}(A / T, X)=0\right\},
\end{gathered}
$$

and

$$
\mathscr{C}=\left\{{ }_{A} X \mid A / T \otimes{ }_{A} X=0\right\} \text {. }
$$

Note that the $\mathscr{T}$-torsion submodule of ${ }_{A} X$ is $X_{t}=r_{X}(T)$ while the $\mathscr{C}$-torsion submodule is $X_{c}=T X$. Furthermore, let

$$
\mathscr{H}=\left\{{ }_{A} X \mid A / T \otimes{ }_{A} M=0 \text { for every submodule } M \text { of } X\right\} \text {. }
$$

Lemma 1.1. $\mathscr{H}$ is a hereditary torsion class and $F(\mathscr{H})=$ $\left\{{ }_{A} I \cong{ }_{A} A \mid(I: a)+T=A\right.$ for all $\left.a \in A\right\}$ where $(I: a)=\{x \in A \mid x a \in I\}$.

Proof. It is left to the reader to check that ${ }_{A} X \in \mathscr{H}$ if and only 
if $x \in T x$ for all $x \in X$. If ${ }_{A} I \in F(\mathscr{X})$, then $A / I \in \mathscr{C}$. Hence $a+$ $I \in T(a+I)$ for all $a \in A$. Thus given $a \in A$ there exists $t \in T$ such that $t a-a=(t-1) a \in I$. Therefore, $t-1 \in(I: a)$, which implies that $1 \in(I: a)+T$.

Conversely, for a left ideal $I$ suppose that $(I: a)+T=A$ for all $a \in A$. Hence given $a \in A$, we have that $1=x+t$ where $x \in(I: a)$ and $t \in T$. Thus $a+I=x(a+I)+t(a+I)$, and so $a+I \in T(a+I)$.

Lemma 1.1 can also be deduced from [9, Lemma 1.1].

LemMa 1.2. For the torsion class $\mathscr{C}$ the following statements are equivalent.

(1) $(A / T)_{A}$ is flat.

(2) $\mathscr{C}$ is hereditary (i.e., $\mathscr{C}=\mathscr{C}$ ).

(3) $\bigcap_{i=1}^{n} l_{A}\left(t_{2}\right)+T=A$ for all $t_{i} \in T$.

(4) $l_{A}(t)+T=A$ for all $t \in T$.

Furthermore, if $\mathscr{C}$ is hereditary, then $F(\mathscr{C})=\left\{{ }_{A} I \cong{ }_{A} A \mid I+T=A\right\}$.

Proof. (1) $\rightarrow(2)$ Take ${ }_{A} X \in \mathscr{C}$. Since $(A / T)_{A}$ is flat $M / T M$ may be viewed as a submodule of $X / T X$. Thus $M / T M=0$ as $X / T X=0$.

$(2) \rightarrow(3)$ Since $T \in \mathscr{C}$ we have that $l_{A}(t) \in F(\mathscr{C})$ for all $t \in T$. Thus $\bigcap_{i=1}^{n} l_{A}\left(t_{2}\right) \in F(\mathscr{C})$ for all $t_{i} \in T$ as $F(\mathscr{C})$ is closed under finite intersections. Hence $\bigcap_{i=1}^{n} l_{A}\left(t_{i}\right)+T=A$ for all $t_{2} \in T$ by Lemma 1.1.

$(3) \rightarrow(4)$ Trivial.

$(4) \rightarrow(1)$ For $t \in T$, by assumption we have that $1=a+t^{\prime}$ where $a \in l_{A}(t)$ and $t^{\prime} \in T$. So $t=a t+t^{\prime} t=t^{\prime} t \in T t$. That $(A / T)_{A}$ is flat now follows by [8, Lemma 4.1].

If $\mathscr{C}$ is hereditary then ${ }_{A} I \in F(\mathscr{C})$ if and only if $T(A / I)=A / I$; i.e., if and only if $I+T=A$.

Since the minimal idempotent ideal $T$ in $F(\mathscr{T})$ is two-sided let

$$
\mathscr{H}^{\prime}=\left\{X_{A} \mid M \otimes{ }_{A} A / T=0 \text { for every submodule } M \text { of } X\right\},
$$

a hereditary torsion class in $\mathscr{A}_{A}$. Let $I$ be a right ideal of $A$ contained in $F\left(\mathscr{H}^{\prime}\right)$. Then $I+T=A$. If $x \in A_{t}=r_{A}(T)$, then $A x=$ $I x+T x=I x \leqq I$. That is, $A_{t}$ is contained in every right ideal in the filter $F\left(\mathscr{C}^{\prime}\right)$. Hence the question arises as to when $A_{t}$ is itself in $F\left(\mathscr{H}^{\prime}\right)$ ?

THEOREM 1.3. Let $\mathscr{T} \leqq{ }_{A} \mathscr{M}$ be a TTF class with associated torsion theories $(\mathscr{F}, \mathscr{F})$ and $(\mathscr{C}, \mathscr{T})$. Using the above notation the following statements are equivalent.

(1) $A_{t} \in F\left(\mathscr{H}^{\prime}\right)$.

(2) $A=A_{t}+A_{c}$.

(3) $X=X_{t}+X_{c}$ for all $X \in_{A} \mathscr{A l}$.

(4) ${ }_{A}\left(A / A_{c}\right)$ is projective. 
(5) $\mathscr{F}$ is closed under homomorphic images.

(6) $\mathscr{F}=\mathscr{H}$.

(7) $\mathscr{F} \subseteq \mathscr{C}$.

Proof. (1) $\rightarrow$ (2) This is immediate from Lemma 1.1.

(2) $\leftrightarrow$ (3) If $A=A_{t}+A_{c}$, then for every ${ }_{A} X$ we have ${ }_{A} X=A_{t} X+$ $A_{c} X \subseteq X_{t}+X_{c}$. The converse is trivial.

$(2) \rightarrow$ (4) This is Lemma 4.10 of [8].

(4) $\rightarrow$ (5) Let ${ }_{A} X \in \mathscr{F}$ and let $\alpha:{ }_{A} X \rightarrow{ }_{A} Y$ be an epimorphism. Since ${ }_{A}(A / T)$ is projective we have an epimorphism $\alpha^{*}: \operatorname{Hom}_{A}(A / T, X) \rightarrow$ $\operatorname{Hom}_{A}(A / T, Y)$. Thus $\operatorname{Hom}_{A}(A / T, Y)=0$; hence $Y \in \mathscr{F}$.

$(5) \rightarrow(6)$ For ${ }_{A} X \in \mathscr{H}$ consider $X_{t} \subseteq X$. Now $T X_{t}=0$; but also $T X_{t}=X_{t}$ since $X \in \mathscr{H}$. Thus $X \in \mathscr{F}$ as $X_{t}=0$.

Conversely, let ${ }_{A} X \in \mathscr{F}$. For $x \in X, A x \in \mathscr{F}$ since $\mathscr{F}$ is closed under submodules. Thus $A x / T x \in \mathscr{F}$ by assumption. But $A x / T x \in \mathscr{T}$ since $T(A x / T x)=0$. Hence $T x=A x$ for all $x \in X$, which implies that $X \in \mathscr{H}$.

$(6) \rightarrow(7)$ Trivial.

$(7) \rightarrow(2)$ By assumption $A / A_{t} \in \mathscr{C}$. Thus $A / A_{t}=T\left(A / A_{t}\right)=T+$ $A_{t} / A_{t}$; i.e., $A_{t}+A_{c}=A$.

$(2) \rightarrow(1)$ For all $t \in T, r_{A}(t)+T=A$ since $A_{t} \subseteq r_{A}(t)$. Thus by Lemma $1.2 F\left(\mathscr{H}^{\prime}\right)=\left\{I_{A} \subseteq A_{A} \mid I+T=A\right\}$. Hence $A_{t} \in F\left(\mathscr{H}^{\prime}\right)$.

REMARK. The equivalence of (2), (3), and (7) was recently shown by Kurata in [7]. He also shows that (5) $\rightarrow(7)$.

A torsion theory $(\mathscr{T}, \mathscr{F})$ is centrally splitting if $\mathscr{T}$ is a TTF class (with associated torsion theories $(\mathscr{T}, \mathscr{F}$ ) and $(\mathscr{C}, \mathscr{T})$ ) and $X=X_{t} \oplus X_{c}$ for all $X \in{ }_{A} \mathscr{M}$. The following corollary characterizing centrally splitting torsion theories is due to Jans [6] and Bernhardt [2].

COROLLARY 1.4. Let $\mathscr{T} \subseteq{ }_{A} \mathscr{C l}$ be a TTF class with associated torsion theories $(\mathscr{T}, \mathscr{F})$ and $(\mathscr{C}, \mathscr{T})$. The following statements are equivalent.

(1) $A=A_{t} \oplus A_{c}$ (ring direct sum).

(2) $X=X_{t} \oplus X_{c}$ for all $X \in{ }_{A} \mathscr{M}$.

(3) $\mathscr{F}=\mathscr{C}$.

(4) $\mathscr{T}$ is stable and $A_{c}$ is a direct summand of $A$.

(5) $\mathscr{F}$ is closed under homomorphic images and $A_{t}$ is a direct summand of $A$.

(6) $A_{c}$ is a ring direct summand of $A$.

Proof. $\mathscr{T}$ being stable (closed under injective hulls) is equivalent to $\mathscr{C}$ being hereditary (see e.g. [2]). Using Lemma 1.2 and Theorem 1.3 conditions (1), (2), (3), (4), and (6) are easily seen to be equivalent 
to the condition that ${ }_{A}\left(A / A_{c}\right)$ is projective and $\left(A / A_{c}\right)_{A}$ is flat. That (1) is equivalent to (5) is easy using Theorem 1.3.

An example of a TTF class $\mathscr{T}$ for which $\mathscr{F}$ is closed under homomorphic images, but $(\mathscr{T}, \mathscr{F})$ is not centrally splitting is given in $\S 2$. See Example 2.5 (i).

In the remainder of this section we investigate the condition that the torsionfree class of a hereditary torsion theory $(\mathscr{T}, \mathscr{F}$ ) be closed under homomorphic images, dropping the condition that $\mathscr{T}$ be a TTF class. Assuming $\mathscr{F}$ is closed under homomorphic images, $\mathscr{F}$ is now a torsion class; i.e., a TTF class. $(\mathscr{T}, \mathscr{F})$ is one torsion theory associated with $\mathscr{F}$. Let $(\mathscr{F}, \mathscr{L})$ be the other. The $\mathscr{T}$-torsion submodule of $A, A_{t}$, is in $F(\mathscr{F})$ since $A / A_{t} \in \mathscr{F}$. Furthermore, if ${ }_{A} I \epsilon$ $F(\mathscr{F})$, then $A_{t} \cap I \in F(\mathscr{F})$, which means that $A / A_{t} \cap I \in \mathscr{F}$. Thus $A_{t} / A_{t} \cap I \in \mathscr{F}$. Since $A_{t} / A_{t} \cap I$ is also in $\mathscr{T}$ we have that $A_{t} / A_{t} \cap$ $I=0$; i.e., $A_{t} \leqq I$. Hence $A_{t}$ is the minimal element of the filter $F(\mathscr{F})$. As before we have that

$$
\begin{gathered}
\mathscr{F}=\left\{{ }_{A} X \mid A_{t} X=0\right\}, \\
\mathscr{L}=\left\{{ }_{A} X \mid \mathrm{Hom}_{A}\left(A / A_{t}, X\right)=0\right\},
\end{gathered}
$$

and

$$
\mathscr{T}=\left\{{ }_{A} X \mid A / A_{t} \otimes{ }_{A} X=0\right\} .
$$

(By Theorem 1.3 if $\mathscr{T}$ is a TTF class then $\mathscr{L}$ is the torsionfree class associated with $\mathscr{C}$.) Since $\mathscr{T}$ is hereditary, $\left(A / A_{t}\right)_{A}$ is flat by Lemma 1.2. Furthermore, $F(\mathscr{T})=\left\{{ }_{A} I \subseteq{ }_{A} A \mid I+A_{t}=A\right\}$.

If in fact $\left(A / A_{t}\right)_{A}$ is projective, then $A=l_{A}\left(A_{t}\right)+A_{t}$ by [8, Lemma 4.10]. Thus $l_{A}\left(A_{t}\right) \in F(\mathscr{T})$. Furthermore, $l_{A}\left(A_{t}\right)$ is the minimal element of $F(\mathscr{T})$ by an argument similar to the one used prior to Theorem 1.3. Hence $\mathscr{T}$ is a TTF class.

Theorem 1.5. For a hereditary torsion theory $(\mathscr{T}, \mathscr{F})$ the following statements are equivalent.

(1) $\mathscr{F}$ is closed under homomorphic images and $\left(A / A_{t}\right)_{A}$ has a projective cover.

(2) $\mathscr{F}$ is closed under homomorphic images and $A_{t}$ is finitely generated as a right $A$-module. jective.

(3) $\mathscr{T}$ is a TTF class where $A_{c}=l_{A}\left(A_{t}\right)$ and $\left(A / A_{t}\right)_{A}$ is pro-

Proof. By our above argument (3) will follow from (1) or (2) if we can show that the flat module $\left(A / A_{t}\right)_{A}$ is projective. In (1) this follows since $\left(A / A_{t}\right)_{A}$ has a projective cover [8, Lemma 1.2]. In (2) this follows by [3, corollary to Proposition 2.2]. 
By Theorem 1.3 (1) and (2) will follow from (3) if we show that $A=A_{t}+A_{c}$. Since $\left(A / A_{t}\right)_{A}$ is projective, $A=A_{t}+l_{A}\left(A_{t}\right)$ by [8, Lemma 4.10]. Thus $A=A_{t}+A_{c}$ by assumption.

Corollary 1.6. (Rutter [10, Proposition 2].) Let $A$ be a semiperfect ring and $(\mathscr{T}, \mathscr{F})$ be a hereditary torsion theory in ${ }_{A} \mathscr{A}$. If $\mathscr{F}$ is closed under homomorphic images, then $\mathscr{T}$ is a TTF class where $A_{c}=l_{A}\left(A_{t}\right)$.

COROLlary 1.7. Let $A$ be a right Noetherian ring and $(\mathscr{T}, \mathscr{F})$ be a hereditary torsion theory in ${ }_{A} \mathscr{A}$. If $\mathscr{F}$ is closed under homomorphic images, then $\mathscr{T}$ is a TTF class where $A_{c}=l_{A}\left(A_{t}\right)$.

2. Projective quasi-generators. In this section $P_{A}$ will be a projective right $A$-module with trace ideal $T=\sum_{f \in \operatorname{Hom}(P, A)} \operatorname{im} f$ and $A$-endomorphism ring $B$. For further information on projective modules and the idempotent trace ideal the reader is referred to [13]. Let $\left.\mathscr{T}={ }_{A} X \mid P \otimes{ }_{A} X=0\right\}$, a hereditary torsion class in ${ }_{A} \mathscr{A C}$. It is easily seen that $\mathscr{T}=\left\{{ }_{A} X \mid T X=0\right\}$ and $F(\mathscr{T})=\left\{{ }_{A} I \subseteq{ }_{A} A \mid T \cong I\right\}$. Hence $\mathscr{T}$ is a TTF class, and the notation of $\S 1$ applies.

Sandomierski [12] has defined an $A$-module ${ }_{A} X\left(X_{A}\right)$ to be $T$-accessible if $T X=X(X T=X)$. Note that $X_{A}$ is $T$-accessible if and only if $X_{A}$ is a homomorphic image of a direct sum of copies of $P_{A}$ [12]. Define an $A$-module $X$ to be strongly $T$-accessible if every submodule of $X$ is $T$-accessible. The class of $T$-accessible (strongly $T$-accessible) left $A$-modules is our class $\mathscr{C}(\mathscr{H})$. From Lemma 1.1 we see that ${ }_{A} X$ is strongly $T$-accessible if and only if $x \in T x$ for all $x \in X$.

We shall call $P_{A}$ a quasi-generator if every $T$-accessible right $A$ module is strongly $T$-accessible. That is, if every submodule of a homomorphic image of a direct sum of copies of $P_{A}$ is itself a homomorphic image of a direct sum of copies of $P_{A}$. This definition is dual to the definition of self-cogenerator given in [11].

THEOREM 2.1. For $P_{A}$ projective with trace ideal $T$ the following statements are equivalent.

(1) $P_{A}$ is a quasi-generator.

(2) $P_{A}$ is strongly T-accessible.

(3) $T_{A}$ is strongly T-accessible.

(4) ${ }_{A}(A / T)$ is flat.

(5) $r_{A}(p)+T=A$ for all $p \in P$.

(6) $r_{A}(t)+T=A$ for all $t \in T$.

Proof. By definition $P_{A}$ is a quasi-generator if and only if the torsion class $\mathscr{C}^{\prime}=\left\{X_{A} \mid X \otimes_{A} A / T=0\right\}$ is hereditary. Thus the equiv- 
alence of (1), (4), and (6) follows by Lemma 1.2. As $\mathscr{H}^{\prime}=$ $\left\{X_{A} \mid M \otimes{ }_{A} A / T=0\right.$ for every submodule $M$ of $\left.X\right\}$ is the class of strongly $T$-accessible right $A$-modules, the equivalence of (2) and (5), and the equivalence of (3) and (6) follows from Lemma 1.1. That (1) implies (2) is by definition.

$(2) \rightarrow(1)$ Let $X_{A}$ be $T$-accessible. Then $X_{A}$ is a homomorphic image of $P_{A}^{I}$, a direct sum of copies of $P_{A}$. Now $P_{A}^{I} \in \mathscr{C}^{\prime}$ as $P_{A} \in \mathscr{H}^{\prime}$ by assumption and $\mathscr{H}^{\prime}$ is closed under direct sums. Thus $X \in \mathscr{H}^{\prime}$ as $\mathscr{H}^{\prime}$ is closed under homomorphic images.

Ware [13] has called a projective module regular if every homomorphic image is flat.

CoRollary 2.2. Let $P_{A}$ be a regular module. Then $P_{A}$ is a quasi-generator. Hence over a regular ring, every projective is a quasi-generator.

Proof. Let $x \in P_{A}$ and consider the exact sequence

$$
0 \longrightarrow x A \longrightarrow P \longrightarrow P / x A \longrightarrow 0 \text {. }
$$

Since $P / x A$ is flat there exists a map $\theta: P_{A} \rightarrow x A$ such that $\theta(x)=x$ [13, Lemma 2.2]. Letting $T$ be the trace ideal of $P_{A}$, we have that $x=\sum_{i=1}^{n} p_{i} t_{i}$ where $p_{i} \in P, t_{i} \in T, i=1,2, \cdots, n$. Hence $x=\theta(x)=$ $\theta\left(\sum_{i=1}^{n} p_{i} t_{i}\right)=\sum_{i=1}^{n} \theta\left(p_{i}\right) t_{i} \in x A T=x T$. Thus $P_{A}$ is strongly $T$-accessible, hence a quasi-generator by Theorem 2.1.

The remainder of the corollary follows from the fact that over a regular ring every projective is regular [13, Example 1, page 238].

In our present framework the question that we asked prior to Theorem 1.3 becomes the following: When is $r_{A}(T)=r_{A}(P)$ strongly $T$-accessible as a right $A$-module?

THEOREM 2.3. Let $P_{A}$ be projective with trace ideal $T$ and $B=$ End $\left(P_{A}\right)$. The follwoing statements are equivalent.

(1) $r_{A}(P)$ is strongly $T$-accessible as a right $A$-module.

(2) $r_{A}(P)+T=A$.

(3) ${ }_{A}(A / T)$ is projective.

(4) $P_{\bar{A}}$ is a generator where $\bar{A}=A / r_{A}(P)$.

(5) $P_{A}$ is a quasi-generator and ${ }_{A} T$ is finitely generated.

(6) $P_{A}$ is a quasi-generator and ${ }_{B} P$ is finitely generated.

Proof. The equivalence of (1), (2), and (3) follows from Theorem 1.3. That (3) is equivalent to (5) follows since $P_{A}$ is a quasi-generator if and only if ${ }_{A}(A / T)$ is flat.

$(2) \leftrightarrow(4) \quad P_{\bar{A}}$ is projective with trace ideal $\bar{T}=T+r_{A}(T) / r_{A}(T)$. 
Thus $r_{A}(P)+T=A$ if and only if $\bar{T}=\bar{A}$; i.e., if and only if $P_{\bar{A}}$ is a generator.

$(4) \rightarrow(6)$ Since $B=$ End $\left(P_{\vec{A}}\right),{ }_{B} P$ is finitely generated. Thus (6) follows by (3).

$(6) \rightarrow(1)$ Since ${ }_{B} P$ is finitely generated we may write $P=B p_{1}+\cdots$ $+B p_{n}$ where $p_{1}, \cdots, p_{n} \in P$. Since $P_{A}$ is a quasi-generator $r_{A}\left(p_{i}\right) \in$ $F\left(\mathscr{H}^{\prime}\right)$ for $i=1, \cdots, n$. Thus $r_{A}(T)=r_{A}(P)=\bigcap_{i=1}^{n} r_{A}\left(p_{i}\right) \in F\left(\mathscr{H}^{\prime}\right)$ since $F\left(\mathscr{H}^{\prime}\right)$ is closed under finite intersections.

REMARK. While $r_{A}(T) \in F\left(\mathscr{H}^{\prime}\right)$ implies that $F\left(\mathscr{H}^{\prime}\right)$ has a minimal element, it is possible for $F\left(\mathscr{H}^{\prime}\right)$ to have a minimal element without $r_{A}(T)$ being contained in $F\left(\mathscr{L}^{\prime}\right)$. Let $A$ be a left perfect ring and let $P_{A}$ be a faithful projective that is not a generator. $F\left(\mathscr{H}^{\prime}\right)$ has a minimal element $L$ [1, Corollary 1.6], but $L \neq r_{A}(T)$. Otherwise $L=0$, which implies that every right $A$-module is torsion; i.e., $P_{A}$ is a generator.

CoRollary 2.4. Let $P_{A}$ be projective with trace ideal $T$ and $B=\operatorname{End}\left(P_{A}\right)$. The following statements are equivalent.

(1) $P_{A}$ is a generator (progenerator).

(2) $P_{A}$ is a (finitely generated) faithful quasi-generator and ${ }_{B} P$ is finitely generated.

(3) $P_{A}$ is (finitely generated) faithful and ${ }_{A}(A / T)$ is projective.

Example 2.5. (i) Let $A$ be the ring of all 2 by 2 upper triangular matrices over a field $K$. Let

$$
e=\left(\begin{array}{ll}
0 & 0 \\
0 & 1
\end{array}\right) \text {. }
$$

Then $P_{A}=e A$ is a projective quasi-generator. ${ }_{B} P$ is finitely generated since ${ }_{B} P=B e$ where $B=e A e$. However, $P_{A}$ is not faithful, so $P_{A}$ is not a generator.

Also, $T=A e A$ is a left direct summand; i.e., ${ }_{A}(A / T)$ is projective. However, $(A / T)_{A}$ is not flat (see Corollary 1.4).

(ii) Let $A=\prod_{i \in I} K_{i}$ where $K_{i}=K$ a field and the index set $I$ is countable infinite. Let $P_{A}=\bigoplus_{i \in I} K_{i}$. We may write $P_{A}=\bigoplus_{i \in I} e_{i} A$ where $e_{i}^{2}=e_{i} \in A$ and $e_{i} A \cong K_{i}$ for $i \in I$. Then $P_{A}$ is projective with trace ideal $T=\bigoplus_{i \in I} e_{i} A$. Since $A$ is a regular ring ${ }_{A}(A / T)$ is flat, and thus $P_{A}$ is a quasi-generator. Furthermore, $P_{A}$ is faithful, but ${ }_{B} P$ is not finitely generated as ${ }_{A} T$ is not finitely generated. Hence $P_{A}$ is not a generator.

REMARK. By Corollary 2.4 (3) if $A$ is a semiperfect ring, then a projective module $P_{A}$ is a generator if and only if $P_{A}$ is a faithful 
quasi-generator. Also, by Corollary 2.4 (2) if $A$ is a regular ring, then a projective module $P_{A}$ is a generator if and only if $P_{A}$ is faithful and ${ }_{B} P$ is finitely generated.

\section{REFERENCES}

1. J. Alin and E. Armendariz, TTF-classes over perfect rings, J. Austral. Math. Soc., 11 (1970), 499-503.

2. R. L. Bernhardt, Splitting hereditary torsion theories over semiperfect rings, Proc. Amer. Math. Soc., 22 (1969), 681-687.

3. S. U. Chase, Direct products of modules, Trans. Amer. Math. Soc., 97 (1960), 457-473.

4. S. E. Dickson, A torsion theory for abelian categories, Trans. Amer. Math. Soc., 121 (1966), 223-235.

5. P. Gabriel, Des catégories abeliennes, Bull. Soc. Math. France, 90 (1962), 323-448.

6. J. P. Jans, Some aspects of torsion, Pacific J. Math., 15 (1965), 1249-1259.

7. Y. Kurata, On an $n$-fold torsion theory in the category ${ }_{R} M$, J. Algebra, 22 (1972), $559-572$.

8. R. W. Miller, Endomorphic rings of finitely generated projective modules, Pacific J. Math., 47 (1973), 199-220.

9. K. Morita, Flat modules, injective modules and quotient rings, Math. Z., 120 (1971), $25-40$.

10. E. Rutter, Torsion theories over semiperfect rings, Proc. Amer. Math. Soc., 34 (1972), 389-395.

11. F. L. Sandomierski, Linearly compact modules and local Morita duality, Proceedings of the Ring Theory Conference, Salt Lake City, Utah, March 1971.

12. Modules over the endomorphism ring of a finitely generated projective module, Proc. Amer. Math. Soc., 31 (1972), 27-31.

13. R. Ware, Endomorphism rings of projective modules, Trans. Amer. Math. Soc., 115 (1971), 233-256.

Received February 14, 1973.

College of William and Mary 



\section{PACIFIC JOURNAL OF MATHEMATICS}

\section{EDITORS}

RICHARD ARENS (Managing Editor)

University of California

Los Angeles, California 90024
J. DUGUNDJI*

Department of Mathematics

University of Southern California

Los Angeles, California 90007

D. Gilbarg and J. Milgram

Stanford University

Stanford, California 94305
University of Washington

Seattle, Washington 98105

ASSOCIATE EDITORS
E. F. BECKENBACH
B. H. NeumanN
F. WoLF
K. YosHIDA

\section{SUPPORTING INSTITUTIONS}

\author{
UNIVERSITY OF BRITISH COLUMBIA \\ CALIFORNIA INSTITUTE OF TECHNOLOGY \\ UNIVERSITY OF CALIFORNIA \\ MONTANA STATE UNIVERSITY \\ UNIVERSITY OF NEVADA \\ NEW MEXICO STATE UNIVERSITY \\ OREGON STATE UNIVERSITY \\ UNIVERSITY OF OREGON \\ OSAKA UNIVERSITY
}

\author{
UNIVERSITY OF SOUTHERN CALIFORNIA \\ STANFORD UNIVERSITY \\ UNIVERSITY OF TOKYO \\ UNIVERSITY OF UTAH \\ WASHINGTON STATE UNIVERSITY \\ UNIVERSITY OF WASHINGTON
* * * *
AMERICAN MATHEMATICAL SOCIETY \\ NAVAL WEAPONS CENTER
}

The Supporting Institutions listed above contribute to the cost of publication of this Journal, but they are not owners or publishers and have no responsibility for its content or policies.

Mathematical papers intended for publication in the Pacific Journal of Mathematics should be in typed form or offset-reproduced, (not dittoed), double spaced with large margins. Underline Greek letters in red, German in green, and script in blue. The first paragraph or two must be capable of being used separately as a synopsis of the entire paper. Items of the bibliography should not be cited there unless absolutely necessary, in which case they must be identified by author and Journal, rather than by item number. Manuscripts, in duplicate if possible, may be sent to any one of the four editors. Please classify according to the scheme of Math. Rev. Index to Vol. 39. All other communications to the editors should be addressed to the managing editor, or Elaine Barth, University of California, Los Angeles, California, 90024.

100 reprints are provided free for each article, only if page charges have been substantially paid. Additional copies may be obtained at cost in multiples of 50 .

The Pacific of Journal Mathematics is issued monthly as of January 1966. Regular subscription rate: $\$ 72.00$ a year (6 Vols., 12 issues). Special rate: $\$ 36.00$ a year to individual members of supporting institutions.

Subscriptions, orders for back numbers, and changes of address should be sent to Pacific Journal of Mathematics, 103 Highland Boulevard, Berkeley, California, 94708.

PUBLISHED BY PACIFIC JOURNAL OF MATHEMATICS, A NON-PROFIT CORPORATION

Printed at Kokusai Bunken Insatsusha (International Academic Printing Co., Ltd.), 270, 3-chome Totsuka-cho, Shinjuku-ku, Tokyo 160, Japan.

* C. R. DePrima California Institute of Technology, Pasadena, CA 91109, will replace J. Dugundji until August 1974.

Copyright (C) 1973 by Pacific Journal of Mathematics

Manufactured and first issued in Japan 


\section{Pacific Journal of Mathematics}

\section{Vol. 51, No. $2 \quad$ December, 1974}

Robert F. V. Anderson, Laplace transform methods in multivariate spectral theory .................................................. 339

William George Bade, Two properties of the Sorgenfrey plane . . . . . . . . . . . . 349

John Robert Baxter and Rafael Van Severen Chacon, Functionals on continuous

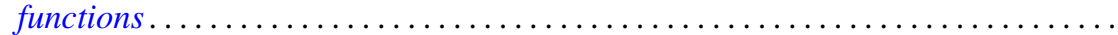

Phillip Wayne Bean, Helly and Radon-type theorems in interval convexity

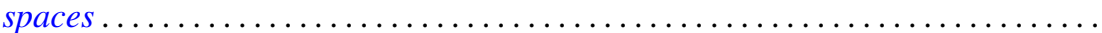

James Robert Boone, On k-quotient mappings $\ldots \ldots \ldots \ldots \ldots \ldots \ldots \ldots \ldots$

Ronald P. Brown, Extended prime spots and quadratic forms . . . . . . . . . . . .

William Hugh Cornish, Crawley's completion of a conditionally upper continuous lattice .............................................

Robert S. Cunningham, On finite left localizations ...................

Robert Jay Daverman, Approximating polyhedra in codimension one spheres

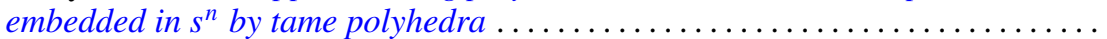

Burton I. Fein, Minimal splitting fields for group representations . . . . . . . . . . . .

Peter Fletcher and Robert Allen McCoy, Conditions under which a connected

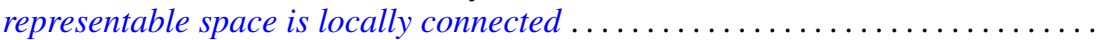

Jonathan Samuel Golan, Topologies on the torsion-theoretic spectrum of a noncommutative ring...

Manfred Gordon and Edward Martin Wilkinson, Determinants of Petrie matrices.

Alfred Peter Hallstrom, A counterexample to a conjecture on an integral condition for determining peak points (counterexample concerning peak points)........

E. R. Heal and Michael Windham, Finitely generated $F$-algebras with applications to Stein manifolds.

Denton Elwood Hewgill, On the eigenvalues of a second order elliptic operator in an unbounded domain ............................

Charles Royal Johnson, The Hadamard product of $A$ and $A^{*}$.

Darrell Conley Kent and Gary Douglas Richardson, Regular completions of Cauchy spaces.

Alan Greenwell Law and Ann L. McKerracher, Sharpened polynomial approximation

Bruce Stephen Lund, Subalgebras of finite codimension in the algebra of analytic functions on a Riemann surface. .

Robert Wilmer Miller, TTF classes and quasi-generators . .

Roberta Mura and Akbar H. Rhemtulla, Solvable groups in which every maximal partial order is isolated ....

Isaac Namioka, Separate continuity and joint continuity...

Alan Saleski, Entropy of self-homeomorphisms of statistical pseudo-metric

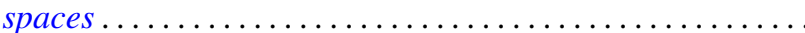

H. A. Seid, Cyclic multiplication operators on $L_{p}$-spaces .....

H. B. Skerry, On matrix maps of entire sequences ............

John Brendan Sullivan, A proof of the finite generation of invariants of a normal

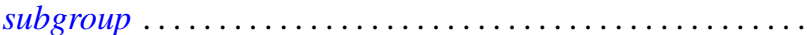

John Griggs Thompson, Nonsolvable finite groups all of whose local subgroups are

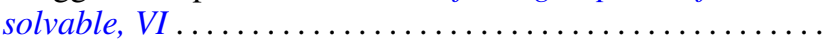

